\title{
On the relationship between coronal waves associated with a CME on 5 March 2000^
}

\author{
D. Tripathi ${ }^{1}$ and N.-E. Raouafi ${ }^{2}$ \\ 1 Department of Applied Mathematics and Theoretical Physics, University of Cambridge, Wilberforce Road, Cambridge, \\ CB3 0WA, UK \\ e-mail: D.Tripathi@damtp.cam.ac.uk \\ 2 National Solar Observatory, 950 North Cherry Avenue, Tucson, AZ 85719, USA \\ e-mail: nraouafi@nso.edu
}

Received 7 February 2007 / Accepted 25 July 2007

\begin{abstract}
Aims. To study the relationship between coronal mass ejection (CME) associated waves.

Methods. Analysis of CME eruption observations on 5 Mar. 2000 recorded by the Large Angle Spectrometric Coronagraph (LASCO), the Ultraviolet Coronagraph Spectrometer (UVCS), and the Extreme-ultraviolet Imaging Telescope (EIT) on board the Solar and Heliospheric Observatory (SOHO).

Results. Images recorded by the LASCO/C2 show a clear deflection and kink in a streamer located eastward of the CME. The kink in the streamer propagated outwards along with the associated CME. No CME material was seen between the bright front of the CME and the streamer. UVCS spectra show large spectral line broadening, Doppler shifts and intensity changes in the O VI ( $\lambda 1032 \& 1037)$ lines. Moreover, intensity enhancements in lines such as Si XII $\lambda 520$ and $\mathrm{Mg}$ X $\lambda 625$ forming at very high temperatures (>2 MK; not often observed in the corona) were also observed. EIT images show the propagation of a wave from the CME source region. The speed of the wave was about $55 \mathrm{~km} \mathrm{~s}^{-1}$ and it propagated predominantly in the North-East direction from the source region. Furthermore, it does not propagate through active regions and coronal holes. The deflection in the streamer recorded in the LASCO/C2 was in the same direction as that of the EIT wave.

Conclusions. Spatial and temporal correlations show that the deflection and the propagation of the kink in the streamer (based on the LASCO data), and plasma heating and spectral line broadening (based on the UVCS data), are basically due to a CME-driven shock wave. The spatial and temporal correlations between the EIT wave and the shock wave provide strong evidence in favor of the interpretation that the EIT waves are indeed the counterpart of CME-driven shock waves in the lower corona. Although, we cannot rule out the possibility that the EIT waves are just a manifestation of the stretching of the field lines due to the outward propagation of the CMEs.
\end{abstract}

Key words. Sun: activity - Sun: corona - Sun: coronal mass ejections (CMEs) - Sun: UV radiation - Sun: chromosphere Sun: filaments

\section{Introduction}

Extreme-ultraviolet (EUV) imaging and spectroscopy provide information on the state of the hot and highly dynamic plasma in the solar corona. While imaging allows the study of plasma dynamics in 2-dimensions projected in the plane of the sky, spectroscopy provides the third component along the line-of-sight (LOS). Using spectroscopic techniques, information about different physical properties such as densities, temperatures, and flow velocities can be obtained based on various spectral lines from different species forming at different temperatures (Mason \& Fossi 1994; Del Zanna et al. 2002).

Coronal mass ejections (CMEs) are one of the most fascinating and intriguing forms of solar activity. They occur when solar plasma threaded with topologically complex magnetic fields are ejected out into the corona and interplanetary medium. They often appear with a three part structure, namely a bright leading edge, a dark cavity, followed by a bright core (Illing \& Hundhausen 1985; Webb 1988; Kahler \& Hundhausen 1992;

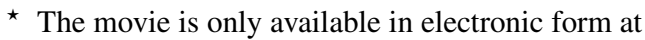
http: //www . aanda.org
Hundhausen 1999). The 3D configuration of such structured CMEs has been studied by Cremades \& Bothmer (2004). Recent technological developments have yielded a steady increase in qualitative and quantitative studies of CMEs and related phenomena. However, the mechanisms responsible for CME initiations and the relationships between different associated phenomena, such as EIT waves (see Thompson et al. 1998), and the existence of CME-driven shock waves (see Hundhausen 1999) are still debated.

CMEs propagate with speed ranging from $50-2500 \mathrm{~km} \mathrm{~s}^{-1}$ with an average speed of $400 \mathrm{~km} \mathrm{~s}^{-1}$ (Gopalswamy et al. 2003; Yashiro et al. 2004). CMEs with speeds greater than the Alfvén speed of the local plasma produce shock waves (see e.g., Priest \& Martens 1984) whose effects can be traced through metric-todecametric solar type II radio bursts (Klassen et al. 2002) and/or emission of very hot lines (compared to the ambient coronal temperatures; Raouafi et al. 2004).

Detection of CME-driven shock waves has been among the most challenging problems in the field of solar physics. The first indirect observation was reported by Hundhausen et al. (1987) and Sime \& Hundhausen (1987) based on the observations 
made by the Solar Maximum Mission (SMM). In this observation, a streamer, which was remotely located with respect to the CME, was deflected in the white light images, without any evident interaction with the CME material and the streamer itself. Further observations of kinking/deflecting streamers were carried out by Sheeley et al. (2000) based on the Large Angle Spectrometric Coronagraph (LASCO; Brueckner et al. 1995) aboard the Solar and Heliospheric Observatory (SOHO; Domingo et al. 1995). Thanks to a better sensitivity, cadence and dynamic range, the LASCO has provided numerous cases for streamer deflection associated with CME eruptions (see e.g., Sheeley et al. 2000).

Sheeley et al. (2000) studied a sample of CME events with super-Alfvénic speeds, which was considered a good proxy for driving shock waves (e.g., Hundhausen 1999), and found that these deflections were indeed a consequence of CME-driven shock, as was previously proposed by Hundhausen et al. (1987) and Sime \& Hundhausen (1987). Later on, however, Kahler (2001) found that the speed of CMEs is rather a poor proxy for the presence of CME-driven shock waves. As far as the direct detection of a CME-driven shock wave by imaging is concerned, we are aware of one event (CME eruption on Apr. 2nd 1999; Vourlidas et al. 2003) where a sharp wave-like feature propagating in the flank of the CME was observed. Vourlidas et al. (2003) also found that this wave-like feature fulfilled the criteria of being a shock wave based on a comparison with an MHD simulation.

CME associated shock waves have also been observed by the UltraViolet Coronagraph Spectrometer (UVCS; Kohl et al. 1995). So far there have been just a few reports of coronal shock waves observed by UVCS, namely events observed on 11 Jun. 1998 (Raymond et al. 2000); 3 Mar. 2002 (Mancuso et al. 2002); 27 Jun. 1999 (Raouafi et al. 2004) and 28 Jun. 2000 (Ciaravella et al. 2005). The spectroscopic observational signatures of a CME-driven shock wave in the corona are spectral line broadening (e.g., O VI $\lambda 1032$ and 1037 lines) and the appearance of very hot lines such as Si XII $\lambda 520$ and Mg X $\lambda 625$ (e.g., Raouafi et al. 2004).

There has been discussion about the solar surface counterpart of the CME-driven shock waves. Since the discovery of the Moreton wave (Moreton 1964), it was thought that these waves were due to the intersection of coronal shock waves (due to flares) with the chromosphere (e.g., Uchida 1968, 1974). Later when Extreme-ultraviolet Imaging Telescope (EIT; Delaboudiniere et al. 1995) waves were observed, they were interpreted as the coronal manifestation of the chromospheric Moreton wave (Thompson et al. 1998, 1999).

Although these waves are now being observed more frequently, thanks to improved instrumentation, the question remains open as to how these different wave phenomena are related to each other. Based on MHD simulations, Chen et al. (2005a,b) showed that the Moreton waves are the surface counter part of the CME driven shock wave. However, EIT waves (Thompson et al. 1998) are the slow moving wave fronts traveling behind the Moreton wave due to the opening of the magnetic flux system. This was also suggested by Delannée (2000); Zhukov \& Auchère (2004). However, this relationship and the nature of EIT waves remain elusive.

SOHO observed a CME on 5 Mar. 2000 with the LASCO/C2 and $\mathrm{C} 3$ telescopes. Fortuitously, this CME was also observed by the UVCS and EIT. This event provides a unique opportunity to establish a relationship between different phenomena, such as the CME associated wave, streamer deflection and the EIT waves.

\section{Observations, data analysis and results}

\subsection{LASCO Observation of a CME, deflecting and kinking streamer}

A white-light CME was observed by the LASCO (Brueckner et al. 1995) C2 and C3 telescopes on 5 Mar. 2000. The LASCO is comprised of three telescopes namely $\mathrm{C} 1$ (5.6 arcsec, $1.1-$ 3.0 $\left.R_{\odot}\right)$, C2 (11.4 arcsec, $\left.2.5-6.0 R_{\odot}\right)$ and C3 (56.0 arcsec, 3.7$\left.30.0 R_{\odot}\right)$. While $\mathrm{C} 1$ failed after a temporary loss of the $\mathrm{SOHO}$ in 1998, C2 and C3 provide continuous observation of the whitelight corona with a time resolution of $20 \mathrm{~min}$ and $54 \mathrm{~min}$, respectively. The CME event studied in this paper first appeared as a three-part structure in the C2 field of view (FOV) at 16:54 UT.

Figure 1 displays the running difference images of the whitelight CME taken by the LASCO/C2 telescope. Running difference images show proper motion of features in the direction of motion (Sheeley et al. 1999). In the running difference whitelight images, if a feature is bright in the absolute images, it appears as a bright new feature, while the dark features represent the traces of those features from the earlier frame, and vice versa. It is easier to identify changes in coronal structures in running difference images due to differential contrast reasons. The threepart structure of the CME is clearly discernible in images as a bright front followed by a dark cavity and a bright core. The bright $\mathrm{CME}$ core is the erupting filament, which has expanded in the LASCO FOV. The leading edge of the CME propagated with a speed of about $860 \mathrm{~km} \mathrm{~s}^{-1}$ (super-Alfvénic) in the FOV of LASCO/C2 and C3. However, the core propagated with a deceleration of $25 \mathrm{~m} \mathrm{~s}^{-2}$ in the LASCO/C2 FOV but reached a constant speed of about $200 \mathrm{~km} \mathrm{~s}^{-1}$ in the LASCO/C3 FOV (Tripathi et al. 2006b).

The arrows in Fig. 1 locate the deflecting and kinking streamer east of the CME. Note the curves in the streamers in the white and black structures, in particular, in the middle panels. Weaker signatures of curving can also be seen in the white features on the far left (see online movie "lasco_eit.gif" 1 ). As can be depicted from the figure, there is no evidence for plasma material seen in between the eastern flank of the CME and the streamer, which can cause such a deflection and kink in the streamer belt. Moreover, the location of the kink in the streamer propagates outward along with the CME. The simultaneous outward propagation of the kink in the streamer and the CME provides strong evidence for the presence of waves. The speed of the propagating kink, measured by tracking the boundary between the bright and dark features from the running difference images, was about $260 \mathrm{~km} \mathrm{~s}^{-1}$ (see, Tripathi \& Raouafi 2006). Note that the speed measured here is in the plane of the sky and thus provides a lower limit to the real speed. Furthermore, the kink in the streamer is likely seen because of a wave phenomenon propagating from the flank of the CME, where the speed of CMEs can be much lower than at the nose (Sheeley et al. 2000). Since the CME propagates with super-Alfvénic speed, it can drive a shock. Moreover, there is no CME material in between the leading edge of the CME and the streamer, which could produce a propagating kink in the streamer. Therefore it is plausible to conclude that the propagating kink was produced due to the shock wave driven by the associated CME.

\footnotetext{
${ }^{1}$ Movie is only available online.
} 

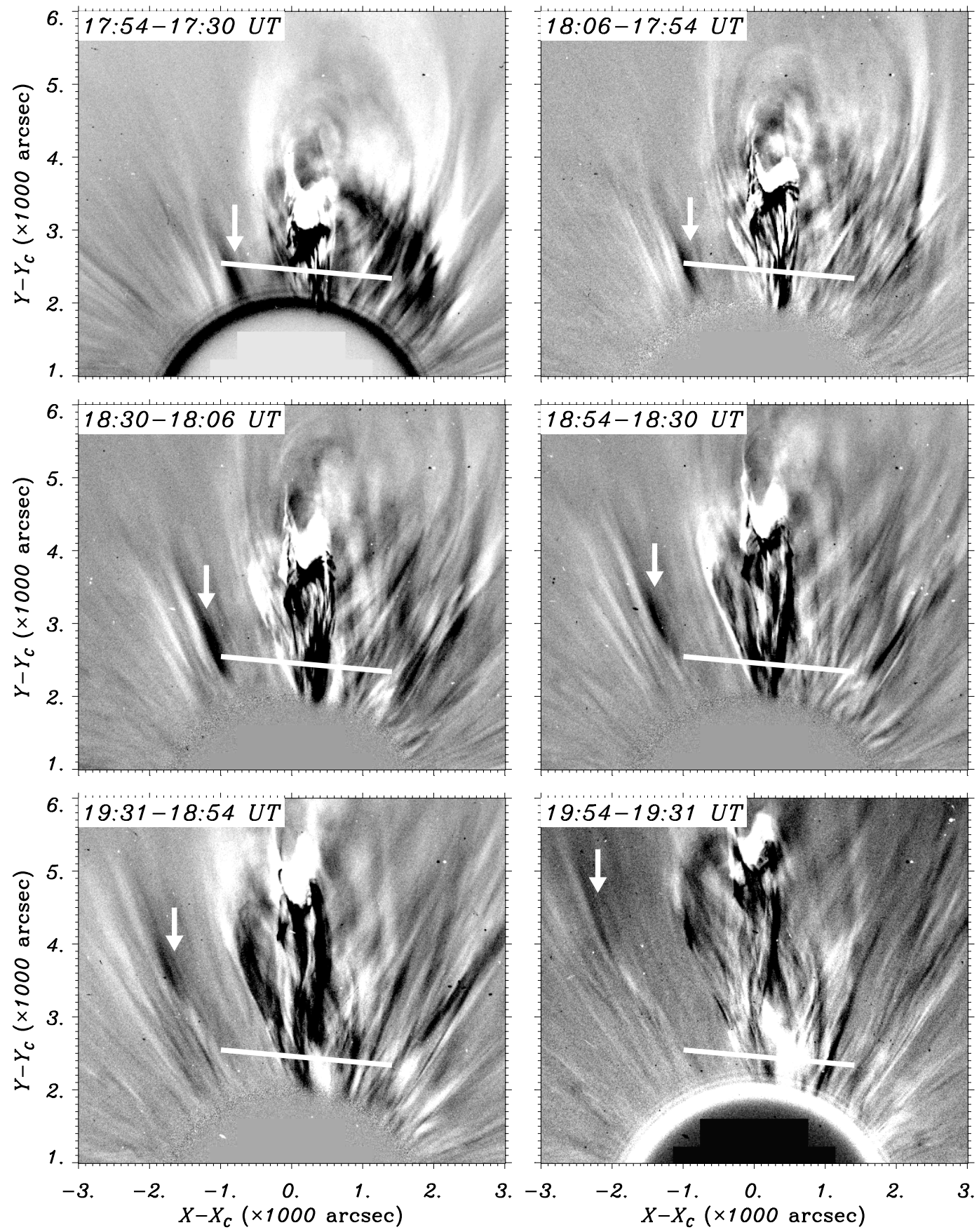

Fig. 1. Running difference white-light images taken from LASCO/C2 telescope. The arrows locate the propagating kink in the streamer. The location of the UVCS slit is given by the white-straight line. $X_{\mathrm{C}}$ and $Y_{\mathrm{C}}$ are the coordinates of the solar disk center.

\subsection{UVCS observations: evidence for a CME-driven shock wave}

UVCS observations of CMEs usually show emission in low to moderate ionization stages, while the emission of higher charged ions becomes fainter or remains unchanged (Raymond 2002). In some cases, UV spectra show emission from higher ionization states that can be interpreted as emission from collisionless shock waves detected in connection with the CME eruption (Raymond et al. 2000; Mancuso et al. 2002) or related to reconnection current sheets (Ciaravella et al. 2005).

The UVCS observational sequence started at 15:58 UT and ended at 20:12 UT, which covers adequately the time evolution of the CME event. The 40 arcmin long UVCS slit was centered at $2.55 R_{\odot}$ from the Sun center at a position angle of $355^{\circ}$ measured counter clockwise from the north pole. The total observational sequence is composed of 80 exposures of $180 \mathrm{~s}$ each. The observation wavelength ranges from $\approx \lambda 1027$ to $\approx \lambda 1042$ for the main channel of the telescope. It includes the strong $\mathrm{O}$ VI doublet lines ( $\lambda 1032$ and $\lambda 1037)$ together with other weaker lines, i.e., Si XII $\lambda 520$ (second order). The redundant channel covers the wavelength interval from $\approx \lambda 1241$ to $\approx \lambda 1252$, which includes the $\operatorname{Mg}$ X $\lambda 625$ line that is also observed in second order. The data set has been calibrated for instrumental effects and wavelength using the most recent release of the UVCS Data Analysis Software (DAS). We concentrate on the first 21 exposures where hot plasma emission is observed and is of relevance to the present paper. 

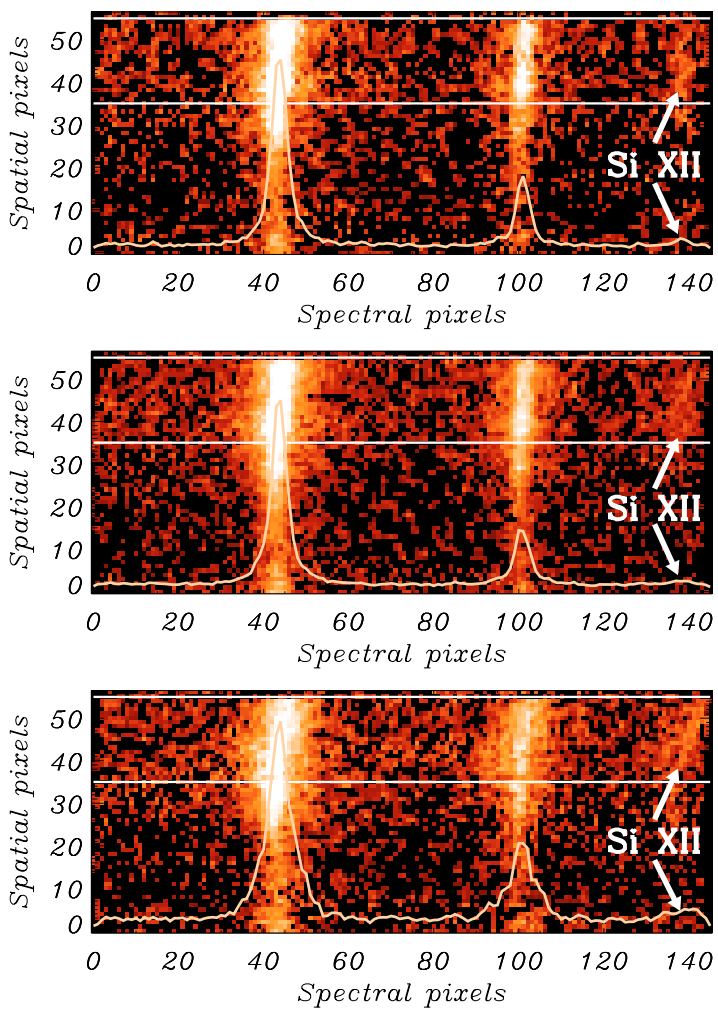

Fig. 2. Averages of the first 21 exposures (top panel: first 5; middle panel: 6 to 15; bottom panel: 16 to 21) of the UVCS observation. The spectral lines shown are the $O$ VI doublet $\lambda 1032$ and $\lambda 1037$ and the weak line of Si XII $\lambda 520$ observed in second order. The spectra overplotted on each panel are the corresponding spatially-binned ones between the two horizontal lines. The focus is on the shape of the profiles.

Figure 2 displays three averaged UVCS exposure sets (top panel: average of exposures from 1 to 5 ; middle panel: average of exposures from 6 to 15; bottom panel: average of exposures from 16 to 21) showing the O VI doublet lines at $\lambda 1032$ and $\lambda 1037$ together with the weak emission of the Si XII $\lambda 520$ line observed in second order. The overplotted curves are the spatially binned spectra between the two horizontal lines. Note that the amplitudes of the overplotted curves are in arbitrary units and should not be confused with the representative of the absolute values.

Due to the relatively high formation temperature ( $>2 \mathrm{MK}$ ) of the Si XII $\lambda 520$ line, this line is rarely observed in the solar corona. Emission in such a line occurs when coronal masses are ejected with high speeds $\left(>600 \mathrm{~km} \mathrm{~s}^{-1}\right)$ that generate coronal shock waves. The latter are responsible for the hot plasma emissions due to compressed gas, as discussed by Raouafi et al. (2004).

The top panel of Fig. 2 shows the O VI line profiles before the CME material has reached the UVCS slit. The O VI line profiles are dominated by a central narrow component. The contribution of an eventual broad component, however, is small. The emission in the Si XII line is weak but remarkable. The line profiles in the middle panel are similar to the previous ones with the difference of an intensity enhancement in the O VI lines. A slight broadening of these lines is also noticeable. In the bottom panel, the O VI lines are dimmed compared to the two previous ones. They are, however, significantly wider, as shown by the overplotted spectra. The emission profile in the Si XII line is also wider and its intensity is relatively enhanced. Doppler shifts are also clear in these lines. The Doppler shifts increase towards the

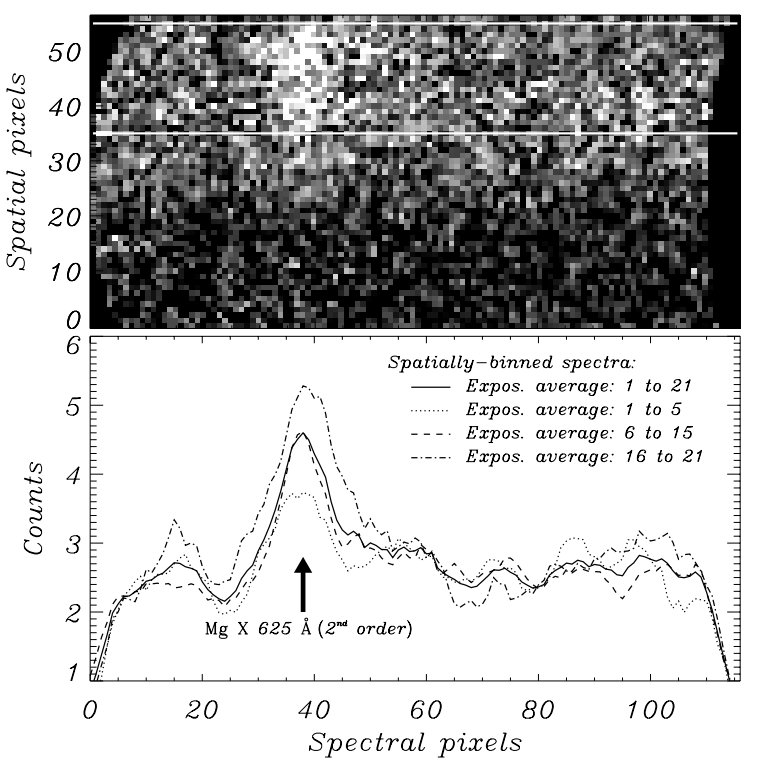

Fig. 3. Top panel: average of the first 21 exposures recorded in the redundant channel of the UVCS. Bottom panel: spatially-binned spectra between the two horizontal lines in the top panel. Spectra are normalized by the number of bins. The meaning of different line-styles is labeled. The strong spectral feature corresponding to the $\operatorname{Mg} \times \lambda 625$ line observation is labeled.

upper end of the slit that is in the North-East direction. In a private communication, Raid Sulaiman (2006) affirmed that these Doppler shifts are real and not due to instrumental effects.

From the bottom panel of Fig. 2 it is evident that the O VI profiles are formed of two components: a broad component representing the emission of hot plasma and a narrow one showing only a small fraction of the original line profiles. The increase in the line profile is very likely due to intense plasma heating as a consequence of a shock wave propagating in front of the associated CME (e.g., Raouafi et al. 2004; Ciaravella et al. 2005). A simple time computation suggested that the shock wave reached the UVCS slit after 16:45 and the heated gas emission lasted till about 17:20 UT, where emission in colder lines is observed. Extra broadening in the O VI lines is noticeable even after the CME cold material has reached the UVCS FOV.

The top panel of Fig. 3 shows the average of the first 21 exposures in the redundant channel, which covers the $\mathrm{Mg} X \lambda 625$. An intensity enhancement around $\lambda 1050$ is clearly evident. This corresponds to the emission of the $\mathrm{Mg}$ X $\lambda 625$ line as observed in the second order of the grating. The bottom panel of the same figure shows the spatially binned spectra between the horizontal lines displayed in the top panel. The solid curve is the spatially binned spectra of all the 21 exposures. The significance of the other curves is explained in the figure's caption. The dot-dashed spectrum shows an intensity enhancement and profile broadening of the $\mathrm{Mg} \mathrm{X}$ line. This took place simultaneously with a change in the profiles of the O VI and Si XII lines.

Figure 4 displays the variations of the $\mathrm{O}$ VI doublet along the slit binned in the spectral direction between spectral bins 35-55 and 90-110. The different curves correspond to the exposures from 16 to 21 . The central peak in the long dashed curve (exposure 21) shows the CME material as it reached the slit. The other features on the right side of the figure correspond to the emission of the plasma heated by the shock wave generated by the CME eruption. These features are slowly drifting towards the right (upper end of the slit that corresponds to the North-East direction). This traces the direction of propagation of the shock 


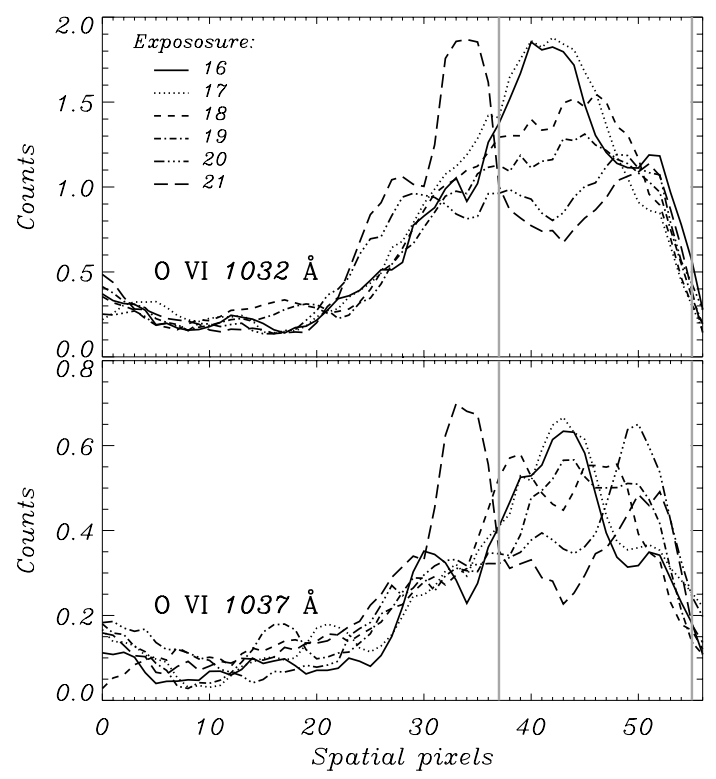

Fig. 4. Intensity variation of the O VI lines along the UVCS slit. The signal is binned in the spectral direction between bins 35-55 for the the $\lambda 1032$ line and 90-110 for the $\lambda 1037$ line. The obtained curves are also smoothed by 5 bins in order to reduce the noise. The meaning of different line-styles is labeled. The vertical gray lines shows the spatial regions used to obtain the spectra shown in Figs. 2 and 3.

wave along the slit. We believe that this is evidence for the physical process causing the intensity variation and line broadening, moving along the slit to the North-East quadrant of the solar corona.

Moreover, we observed the intensity dimming in the upper section of the slit of the the O VI $\lambda 1032$ line from one exposure to the next, where that of the $\lambda 1037$ line changes quite differently. It becomes enhanced, in particular, in the exposure number 20. This provides evidence for the acceleration of the $\mathrm{O}$ VI ions that leads the $\lambda 1032$ line to run out of resonance and the $\lambda 1037$ line to get optically pumped by the chromospheric lines of C II.

\subsection{EIT observations: EIT wave}

The EIT observes the solar corona in four different wavelength channels. Solar images at $\lambda 195$ are recorded regularly with a cadence of 12 minutes and are used for the present study. Usually the features observed in the $\lambda 195$ channel are dominated by Fe XII emission forming at $1.5 \mathrm{MK}$, however during flares the dominant emission in the EIT images are due to an Fe XXIV $\lambda 192$ line that forms at a much higher temperature (about $20 \mathrm{MK}$; Tripathi et al. 2006a).

Figure 5 displays the running difference images taken by EIT at $\lambda 195$. The filament slowly rose and erupted around 15:46 UT. The EIT wave associated with this eruption first appeared at 16:10 UT and disappeared at 16:58 UT.

The EIT wave was only seen propagating towards the North-East. No counter-part movements towards the South-West were detected. The propagating wave-front did not traverse through the coronal hole, which was located in the North-East of the source region, similar to the observations presented by Thompson et al. (1999). Figure 6 displays the distance-time profile of the wave measured based on the running difference of EIT images. The dashed line represents a linear fit to the data points, which was used to derive the speed of the EIT wave. The average speed of the propagation was about $55 \mathrm{~km} \mathrm{~s}^{-1}$. The speed of the EIT wave obtained here is smaller than those reported earlier by Thompson et al. (1999); Klassen et al. (2000). Note that no correction for the projection effect was performed while measuring the speed. Except for the speed being much lower, other characteristics are similar to those of the EIT waves.

\section{Discussion}

The information obtained from the spectral data recorded by the UVCS instrument provides strong evidence that the March 5, 2000 CME was accompanied by a shock wave. However, the question remains as to whether this shock wave is linked to the deflection/kink observed in the remote streamer detected in the LASCO images, and to EIT waves. Spatial and temporal correlations of the different observed wave phenomena would help us resolve this issue.

Based on the intensity changes and spectral line broadening in the O VI $\lambda 1032$ and $\lambda 1037$ lines and also the appearances of the hot lines such as Si XII $\lambda 520$ and $\mathrm{Mg} \mathrm{X} \lambda 625$, it was estimated that the shock wave would have reached the UVCS slit (placed at $2.55 R_{\odot}$ ) at around 16:45 UT. However, the first deflection in the streamer based on the LASCO image was seen much later at around 17:30 UT at a height (projected in the plane of sky) of about $2.5 R_{\odot}$ from the disk center. On one hand since the CME is fast enough to generate a shock wave and if we take into account the analysis performed by Sheeley et al. (2000), we conclude that the kink was observational evidence for the CME-driven shock wave. On the other hand, based on the temporal correlation, it is evident that the shock wave did not propagate with isotropic speed. This difference in the speed can be explained by different speeds in different parts of CMEs.

The question now is whether the EIT wave observed in the low corona (on the solar surface) could be associated with the CME driven shock wave observed by the LASCO (streamer deflection/kink) and in UVCS spectra (as the intensity enhancement and spectral line broadening). The EIT wave was first observed at 16:10 UT and it disappeared from the EIT FOV at around 16:58 UT. The UVCS observations show that the arrival time of the shock at the UVCS slit (at $2.55 R_{\odot}$ ) was about 16:45 UT. The speed of the EIT wave is about $55 \mathrm{~km} \mathrm{~s}^{-1}$, which is much slower than the expected shock wave speed that is of the order of Alfvénic speed based on theoretical modeling (e.g., Uchida 1968; Chen et al. 2005b). Note that no correction for a geometrical projection effect was performed while estimating the speed of EIT waves.

The three features observed by EIT, LASCO and UVCS are generated by the same source, propagate in the same direction and have good time and spatial correlations. The only apparent problem resides in the propagation speed. However, the three wave like structures do not share the same propagation medium and physical conditions. One of these is the space and its characteristics. The speed of Alfvén waves is given by $V_{\mathrm{A}}=\frac{B}{\sqrt{4 \pi \rho}}$, where $B$ is the magnetic field strength and $\rho$ is the density. Depending on models, the density drops by 3 to 4 orders of magnitude or more between the very low corona and 2.0-3.0 $R_{\odot}$, where the magnetic field drops by an order of magnitude or more (assuming that the coronal field is potential and thus drops as $r^{-2}$; see Altschuler \& Newkirk 1969). Meanwhile the high corona is increasingly less dense with increasing altitude and the corresponding Alfvén speed tends to increase. This is not the case in the lower corona where the plasma density is higher and thus is 

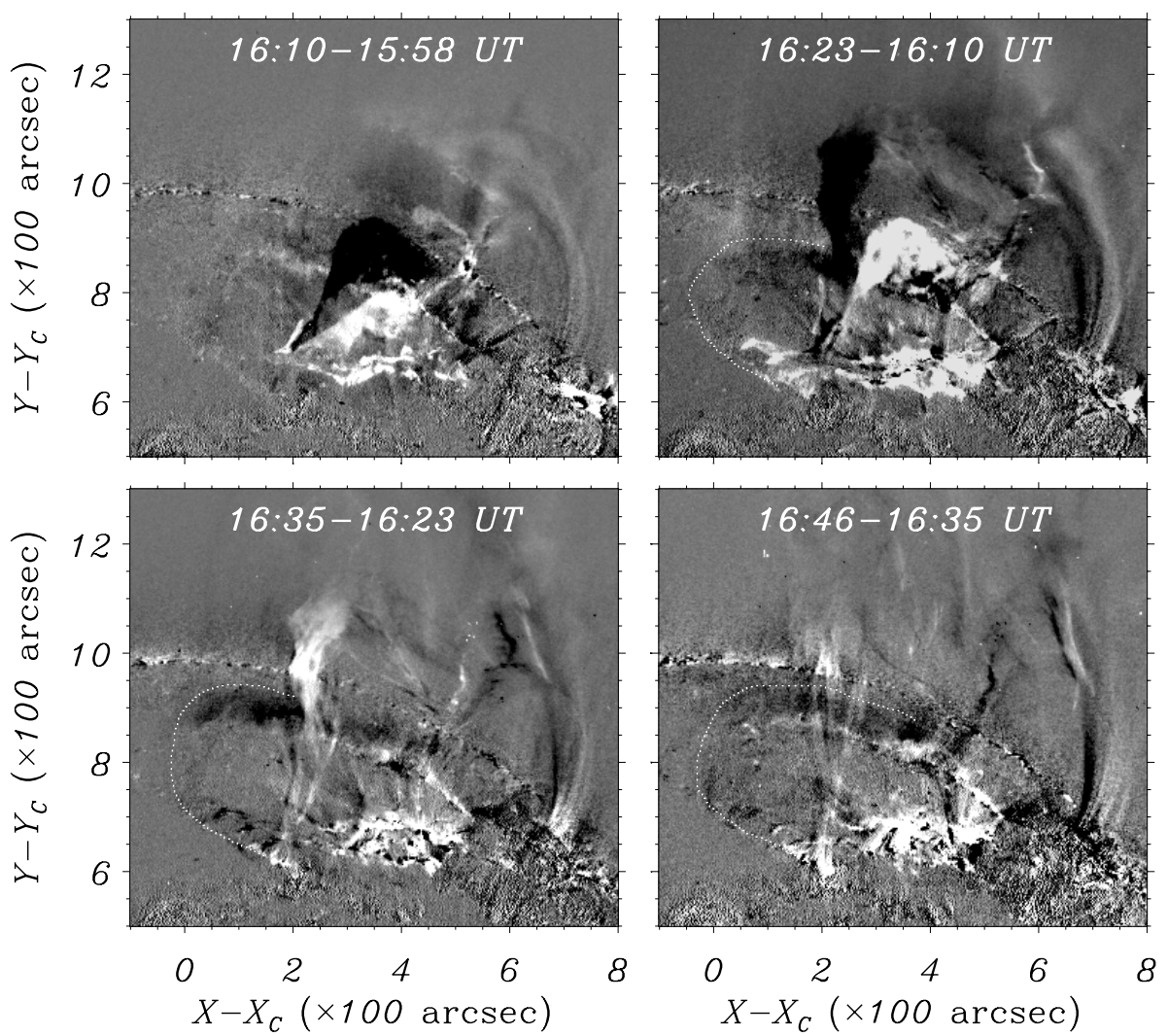

Fig. 5. EIT running difference images showing the EIT waves. The dotted curves demark the external edge of the EIT wavefront.

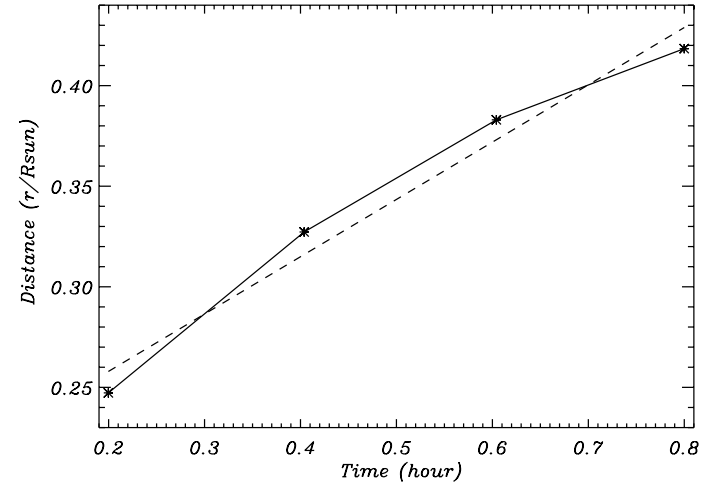

Fig. 6. Distance-time diagram of the EIT waves measured based on running difference images. The asterisks and the solid line represent the actual data points. The dashed line is a linear fit to the data points.

characterized by a smaller Alfvén speed. This could explain the difference in speed.

Another parameter is the propagation direction of the waves. The CME-driven shock wave is mainly propagating parallel to the magnetic field lines. However, the wave propagating on the solar disk does not share this property and propagates across. It encounters different magnetic structures that may allow for energy leakage, which consequently might dampen and slow down the wave (see for instance De Pontieu et al. (2004) on energy leak of the acoustic oscillation p modes through flux tubes with high inclinations to the chromosphere, which contributes to the heating of this layer). A good illustration for this is given by active regions through which EIT waves could not propagate and also across polar holes. We think that energy carried by the wave is progressively leaked to different magnetic structures, which may heat and accelerate the plasma in these structures. This interpretation needs to be carried further and deeper through additional analysis of other observational examples and also by numerical simulations.

However, we cannot rule out that EIT wave-like features result from opening of the magnetic flux system due to the expulsion of a CME, as suggested by Chen et al. (2005b,a) based on MHD modeling and by Zhukov \& Auchère (2004); Delannée (2000) based on observations. Unfortunately, we did not have $\mathrm{H}_{\alpha}$ high cadence observations for this event, which prevented a comparison with a chromospheric moreton wave (if present).

\section{Summary and conclusions}

We have identified different coronal waves associated with a CME observed on March 5, 2000 and studied their relationship. The CME event was recorded by different instruments aboard SOHO (EIT, LASCO and UVCS). The summary of the observational results and conclusions are as follows:

- The LASCO white-light images show a deflection in a remote streamer, located at an approximate angular position of $10-15^{\circ}$ counterclockwise from the north pole, with respect to the CME site. The deflection was observed in the form of a kink that propagated along with the CME with a speed of about $260 \mathrm{~km} \mathrm{~s}^{-1}$ (projected on the plane of the sky). The white-light images show no evidence of the presence of any CME material that could cause such an effect. Furthermore, the CME was traveling at $860 \mathrm{~km} \mathrm{~s}^{-1}$ (superAlfvénic speed), which is sufficient to drive a shock wave. Therefore, as Sheeley et al. (2000) have suggested, this propagating kink in the streamer provides strong evidence for a CME-driven shock wave in the corona. 
- The spectra recorded by UVCS show excessive broadening in O VI $\lambda 1032$ and $\lambda 1037$ lines. Moreover, the spectra also reveal broadenings and intensity enhancements in the hot lines of Si XII $\lambda 520$ and $\mathrm{Mg}$ X $\lambda 625$, which are rarely observed in the corona at such heights $\left(2.0-3.0 R_{\odot}\right)$ because of their very high formation temperatures. These are clear evidence for a CME-driven shock wave (Raymond et al. 2000; Raouafi et al. 2004; Ciaravella et al. 2005). In addition, the analysis of the intensity modulation along the slit reveals the propagation direction of the wave which, is the same as that of the streamer deflection.

- EIT difference images show evidence for an EIT wave front propagating North-East with speed of about $55 \mathrm{~km} \mathrm{~s}^{-1}$. Note that taking into account the solar disk shape would significantly magnify the value of the speed. The wave-front did not traverse through the region of the open field line (polar hole), which was located in the North-East direction of the source region, satisfying the properties of EIT waves (Thompson et al. 1999). The EIT wave first appeared at 16:10 UT and disappeared at around 16:58 UT from the EIT FOV. The spatial and temporal correlation between the EIT wave and the CME-driven shock (observed by LASCO and UVCS) wave provide strong evidence in favor of the interpretation that the EIT waves are indeed a counterpart of the CME-driven shock wave in the lower corona.

The March 5, 2000 event is indeed an interesting and unique (to the best of our knowledge) case for studying the relationship between different coronal waves. However, we note that this is just one event study and we intend to extend this study in the future. The ideal data set required for these kind of study would $\mathrm{H}_{\alpha}$, EUV images and spectroscopic data for CME with very high temporal resolution. We expect that the imaging and spectroscopic observations taken by Hinode, STEREO and SOHO, combined with ground based observations, would enable us to understand the phenomena better.

Acknowledgements. We thank the referee Dr. Volker Bothmer for his constructive comments which improved the quality of the manuscript. DT acknowledges the funding from STFC and NER is supported by NSO and by NASA grant NNH05AA12I. We thank the SOHO-EIT, LASCO, and UVCS team for providing the data. SoHO is a project of international collaboration between ESA and NASA. The NSO is operated by the Association of Universities for Research in Astronomy (AURA), Inc. under cooperative agreement with the National Science Foundation (NSF).

\section{References}

Altschuler, M. D., \& Newkirk, G. J. 1969, Sol. Phys., 9, 131

Brueckner, G. E., Howard, R. A., Koomen, M. J., et al. 1995, Sol. Phys., 162, 357

Chen, P. F., Ding, M. D., \& Fang, C. 2005a, Space Sci. Rev., 121, 201

Chen, P. F., Fang, C., \& Shibata, K. 2005b, ApJ, 622, 1202

Ciaravella, A., Raymond, J. C., Kahler, S. W., Vourlidas, A., \& Li, J. 2005, ApJ, 621,1121

Cremades, H., \& Bothmer, V. 2004, A\&A, 422, 307

De Pontieu, B., Erdélyi, R., \& James, S. P. 2004, Nature, 430, 536

Del Zanna, G., Landini, M., \& Mason, H. E. 2002, A\&A, 385, 968

Delaboudiniere, J.-P., Artzner, G. E., Brunaud, J., et al. 1995, Sol. Phys., 162, 291

Delannée, C. 2000, ApJ, 545, 512

Domingo, V., Fleck, B., \& Poland, A. I. 1995, Space Sci. Rev., 72, 81

Gopalswamy, N., Lara, A., Yashiro, S., Nunes, S., \& Howard, R. A. 2003, in Solar Variability as an Input to the Earth's Environment, ed. A. Wilson, ESA SP-535, 403

Hundhausen, A. 1999, in The many faces of the sun: a summary of the results from NASA's Solar Maximum Mission, ed. K. T. Strong, J. L. R. Saba, B. M. Haisch, \& J. T. Schmelz, 143

Hundhausen, A. J., Holzer, T. E., \& Low, B. C. 1987, J. Geophys. Res., 92, 11173

Illing, R. M. E., \& Hundhausen, A. J. 1985, J. Geophys. Res., 90, 275

Kahler, S. W. 2001, J. Geophys. Res., 106, 20947

Kahler, S. W., \& Hundhausen, A. J. 1992, J. Geophys. Res., 97, 1619

Klassen, A., Aurass, H., Mann, G., \& Thompson, B. J. 2000, A\&AS, 141, 357

Klassen, A., Bothmer, V., Mann, G., et al. 2002, A\&A, 385, 1078

Kohl, J. L., Esser, R., Gardner, L. D., et al. 1995, Sol. Phys., 162, 313

Mancuso, S., Raymond, J. C., Kohl, J., et al. 2002, A\&A, 383, 267

Mason, H. E., \& Fossi, B. C. M. 1994, A\&ARv, 6, 123

Moreton, G. F. 1964, AJ, 69, 145

Priest, E. R., \& Martens, P. C. H. 1984, Ruimtevaart, 33, 119

Raouafi, N.-E., Mancuso, S., Solanki, S. K., et al. 2004, A\&A, 424, 1039

Raymond, J. C. 2002, in From Solar Min to Max: Half a Solar Cycle with SOHO, ed. A. Wilson, ESA SP-508, 421

Raymond, J. C., Thompson, B. J., St. Cyr, O. C., et al. 2000, Geophys. Res. Lett., 27, 1439

Sheeley, N. R., Walters, J. H., Wang, Y.-M., \& Howard, R. A. 1999, J. Geophys. Res., 104, 24739

Sheeley, N. R., Hakala, W. N., \& Wang, Y.-M. 2000, J. Geophys. Res., 105, 5081

Sime, D. G., \& Hundhausen, A. J. 1987, J. Geophys. Res., 92, 1049

Thompson, B. J., Plunkett, S. P., Gurman, J. B., et al. 1998, Geophys. Res. Lett., 25,2465

Thompson, B. J., Gurman, J. B., Neupert, W. M., et al. 1999, ApJ, 517, L151

Tripathi, D. \& Raouafi, N.-E. 2006, in ESA SP-617, SOHO-17, 10 Years of SOHO and Beyond

Tripathi, D., Del Zanna, G., Mason, H. E., \& Chifor, C. 2006a, A\&A, 460, L53

Tripathi, D., Solanki, S. K., Schwenn, R., et al. 2006b, A\&A, 449, 369

Uchida, Y. 1968, Sol. Phys., 4, 30

Uchida, Y. 1974, Sol. Phys., 39, 431

Vourlidas, A., Wu, S. T., Wang, A. H., Subramanian, P., \& Howard, R. A. 2003, ApJ, 598, 1392

Webb, D. F. 1988, J. Geophys. Res., 93, 1749

Yashiro, S., Gopalswamy, N., Michalek, G., et al. 2004, J. Geophys. Res. (Space Phys.), 109, 7105

Zhukov, A. N., \& Auchère, F. 2004, A\&A, 427, 705 